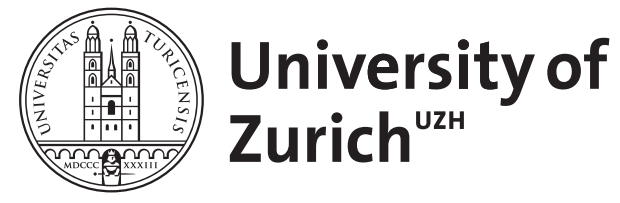
Archive

University of Zurich

University Library

Strickhofstrasse 39

CH-8057 Zurich

www.zora.uzh.ch

Year: 2005

Physician Competency? Teaching Old Dogs New Tricks

Clavien, P A ; Nahrwold, D ; Soper, N ; Bass, B

DOI: https://doi.org/10.1016/j.gassur.2005.01.289

Posted at the Zurich Open Repository and Archive, University of Zurich

ZORA URL: https://doi.org/10.5167/uzh-156637

Journal Article

Published Version

Originally published at:

Clavien, P A; Nahrwold, D; Soper, N; Bass, B (2005). Physician Competency? Teaching Old Dogs New

Tricks. Journal of Gastrointestinal Surgery, 9(5):625-632.

DOI: https://doi.org/10.1016/j.gassur.2005.01.289 


\title{
Physician Competency? Teaching Old Dogs New Tricks
}

\author{
Pierre-Alain Clavien, M.D., Ph.D., David L. Nahrwold, M.D., Nathaniel F. Soper, M.D., \\ Barbara Lee Bass, M.D.
}

KEY wORDs: Physician competency

\section{INTRODUCTION Pierre-Alain Clavien, M.D., Ph.D.}

The public policy committee of the Society for Surgery of the Alimentary Tract (SSAT) held a panel on "Physician Competency" during Digestive Disease Week in New Orleans on May 18, 2004. Developing and assessing physician competencies, particularly surgeon competencies, is a challenge and a subject of many discussions worldwide. The goal of surgical training in any system is to produce competent professionals capable of meeting the health care needs of the society. A surgeon must learn to operate safely and skillfully. The traditional way of teaching has been in the operating room, is based on an exemplary role model, and is monitored through subjective assessments. "See one, do one, teach one" has been the adage of many generations. The operating room is no longer the ideal learning environment because of (a) increasing time constraints, (b) cost, stress, and ethical considerations, (c) hours and shift restrictions for residents, (d) a shift from inpatient to ambulatory surgery, (e) the use of more complex (laparoscopic) procedures, and (f) the public's attention. These constraints have resulted in a shift towards a more objective measurement of outcome and surgeon abilities, and, thereby, decreased interaction between residents and senior role models.
What makes a surgeon competent? Certificates and diplomas are still important testimonies of achievements, but will the future rely on new teaching tools, new routines, and continuous assessments? In this symposium, David Nahrwold attempts to define "What is Competence?" Nat Soper provides insights into "Teaching Old Dogs New Tricks," and finally Barbara Bass covers the topic of "Measuring and Maintaining Competency."

\section{WHAT IS COMPETENCE? David L. Nahrwold, M.D.}

The purpose of this section is to review the genesis of the competence movement in medicine and describe competence as it applies to the medical profession. Previous reviews of this subject have been published elsewhere. ${ }^{1-4}$

Our society is pervaded by a fixation on quality. The business and industrial community recognizes that high-quality products and services are essential to compete in our global economy, using techniques and concepts such as zero defects and six sigma. Accordingly, the public has expectations that providers of services, including mechanics, hairdressers, lawyers, and physicians, will be competent.

Presented at the SSAT Public Policy Symposium held during the Forty-Fifth Annual Meeting of The Society for Surgery of the Alimentary Tract, New Orleans, Louisiana, May 15-19, 2004.

From the Department of Surgery, University Hospital Zurich (P.A.C.), Zurich, Switzerland; Department of Surgery, Feinberg School of Medicine (D.L.N., N.J.S.), Northwestern University, Chicago, Illinois; and Department of Surgery, University of Maryland School of Medicine (B.L.B.), Baltimore, Maryland.

Reprint requests: Pierre-A. Clavien, M.D., Ph.D., University Hospital of Zurich, Department of Visceral and Transplant Surgery, Raemistrasse 100, 8091 Zurich, Switzerland. e-mail: clavien@chir.unizh.ch 
Webster's New World College Dictionary ${ }^{5}$ defines competence and competent in a typical manner: "com-pe-tence $\mathrm{n}$. Condition or quality of being competent." "com-pe-tent adj. Well qualified; capable; fit (a competent doctor)." Thus, although competence and the competent individual are defined, the competent physician is not.

The public wants competent physicians. Variations in care, poor service, and preventable medical errors have eroded confidence in the competence of physicians in recent years, even to the extent that the professional privilege of self-regulation is endangered. To restore the public trust, physicians must evaluate their competence as a profession and as individual practitioners within the health care system. Individually and as a profession, physicians must provide the public with evidence of their competence.

In the United States, the profession and individual physicians can demonstrate their competence to the public through certification by a specialty board that is a member of the American Board of Medical Specialties (ABMS). Currently, approximately 90\% of physicians in the Unites States are certified by at least one ABMS member board. Physicians view their certification as validation of their education and training and confirmation that they possess the requisite knowledge to practice their specialty. Hospitals, insurance companies, and health plans value certification as an indicator of quality, for most require board certification for medical staff or physician panel membership and for awarding clinical privileges. The public has been educated to believe that certification is an important, distinguishing credential for physicians. Thus, certification has earned credibility among physicians, health care organizations, and the public.

ABMS, the umbrella organization for the 24 medical specialty boards, consists of representative of the boards who set standards, share information, and monitor the performance of the boards (Table 1). In the late 1990s, ABMS, although satisfied with the rigor and process of initial certification, began to be concerned that recertification was insufficient evidence that a physician was delivering quality care. Most boards required diplomates to recertify every 10 years by passing an examination. ABMS and its member boards realized that the turnover of much knowledge was faster than every 10 years, and that although an examination measured factual knowledge and some aspects of medical judgment, it was not always relevant to the individual physician's practice. Although an essential element, an examination for medical knowledge does not connote competence or adequately measure the broader range of attributes the public desires in their physicians. In 1998 the
Table 1. Member boards of the American Board of Medical Specialties (ABMS)

- Allergy and Immunology

- Anesthesiology

- Colon and Rectal Surgery

- Dermatology

- Emergency Medicine

- Family Practice

- Internal Medicine

- Medical Genetics

- Neurological Surgery

- Nuclear Medicine

- Obstetric and Gynecology

- Ophthalmology

- Orthopedic surgery

- Otolaryngology

- Pathology

- Pediatrics

- Physical Medicine and Rehabilitation

- Plastic Surgery

- Preventative Medicine

- Psychiatry and Neurology

- Radiology

- Surgery

- Thoracic Surgery

- Urology

Bold indicates that a surgical board is available.

ABMS appointed its Task Force on Competence, which attempted to define competence and to change the process of recertification to more adequately measure the characteristics required of contemporary physicians to competently practice their specialties.

The task force found that it could not define physician competence, and recognized that no appellation could warrant that a physician was competent at all times for all aspects of his or her practice. Nevertheless, the task force was able to describe the competent physician, and did so as follows: "The competent physician possesses the medical knowledge, judgment, professionalism, and clinical and communications skills to provide high-quality patient care. Patient care encompasses the promotion of health, prevention of disease, and diagnosis, treatment, and management of medical conditions with compassion and respect for patients and their families. Maintenance of competence should be demonstrated throughout the physician's career by evidence of lifelong learning and ongoing improvement of practice." The task force then began to identify the competencies embedded in this description.

Concomitantly, the Accreditation Council on Graduate Medical Education (ACGME) was studying physician competence and obtaining the expertise and 
Table 2. Six general competencies according to Accreditation Council on Graduate Medical Education and the American Board of Medical Specialties

1. Medical knowledge

2. Patient care

3. Interpersonal and communication skills

4. Professionalism

5. Practice-based learning and improvement

6. Systems-based practice

opinions of educators, practicing physicians, patients, health care experts, and opinion leaders to determine their expectations of physicians. The ACGME categorized the many desired attributes of physicians into six general competencies that collectively describe the competent, contemporary physician and are embedded in the description of the competent physician (Table 2). Together, the ACGME and the ABMS adopted the six general competencies as attributes that should be taught to residents and should be displayed by residents as well as physicians throughout their careers.

Subsequently, ABMS adopted a new paradigm for the evaluation of practicing physicians, moving from recertification every 10 years to maintenance of certification, in which diplomates will "maintain" their initial certification by ongoing, periodic demonstration of the six general competencies throughout their practicing lives. Diplomates also will be required to meet other requirements of their boards. The maintenance of certification programs of ABMS member boards are designed to help diplomates improve their practices. The improvement paradigm requires the diplomate to accumulate data about his or her performance and to improve it through a variety of learning modalities. It is the expectation of ABMS and its member boards that initial certification and maintenance of certification will serve as an indicator of quality and be accepted as such by organizations that monitor quality of care, health plans, and the public.

\section{TRAINING IN LAPAROSCOPIC SURGERY: "TEACHING OLD DOGS NEW TRICKS" Nathaniel J. Soper, M.D.}

Laparoscopic operations can be broken down into two general types of procedures-basic laparoscopy (e.g., diagnostic laparoscopy, cholecystectomy, and appendectomy), and advanced laparoscopy, which requires two-handed manipulation, alternative viewing angles of the field, as well as suturing and other skills. The "advanced" category includes essentially everything other than the above "basic" procedures. As opposed to the early days of laparoscopic general surgery, residents now are taught basic laparoscopic techniques during their training period.

However, learning minimally invasive surgery (MIS) is different than learning "open" surgery, and MIS training in residency is very uneven. ${ }^{6}$ The learning curve for the majority of advanced procedures is greater than the number of cases available in most residencies. ${ }^{7}$ Furthermore, it is more difficult as an attending surgeon to "control" residents during performance of MIS. To add to the difficulty of teaching MIS, operating room time is limited and expensive. Furthermore, MIS itself is significantly different than open surgery. A trainee must learn the physiology of the pneumoperitoneum and various techniques to access the abdominal cavity. Using a video screen, vision is monocular with limited depth perception. Furthermore, there is decreased haptic feedback using laparoscopic instrumentation. The long instruments amplify tremor and the image is magnified to $10 \times$. There is also a fulcrum effect whereby the tip of the laparoscopic instrument moves in the opposite direction of its handle. Finally, the fixed access through the trocar limits the degrees of freedom of the instruments.

There are several venues for teaching fundamental laparoscopic skills, such as recreating three-dimensional depth relationships and navigation of instruments in space. These include the animate laboratory, the inanimate laboratory, and the operating room. The animate laboratory employs cadaveric or large animal models for training. These types of models are very expensive and the use of either cadavers or animals may be a sensitive issue in various areas. Inanimate laboratories have been used to good effect. The training devices currently available include so-called video trainers (an opaque box with trocars allowing access to the interior wherein various physical materials can be manipulated in specific exercises) and virtual reality (VR) simulators. VR trainers allow rudimentary manipulation of instruments and tissues. VR trainers are currently somewhat "cartoonish" with suboptimal tissue interactions and haptics, but the simulator quality is improving rapidly. Finally, several procedure-specific rubber models are being developed for use with laparoscopic trainers. The final location for learning minimally invasive surgery is in the operating room-one of Halsted's training tenets was that the operating theater would be the surgeon's classroom. However, it is increasingly difficult to teach technical skills in the operating room because of expense, the limited resource of time in 
the operating room, and concerns regarding medical errors caused by novice trainees.

Inanimate laboratory training thus assumes an important role in teaching MIS. Trainees who practice in a video trainer improve their performance of the trainerspecific skills. A plateau seems to be reached after 30 to 35 repetitions, and the least experienced individuals improve the most. ${ }^{6,8}$ However, quantifying skills in a video trainer is problematic, generally requiring a proctor to be present if something more than time of performance is to be included in the scoring. Two reports have shown that practice in a video trainer improves in vivo performance by trainees during subsequent laparoscopic cholecystectomy. 6,8

The group from McGill University has developed the McGill Inanimate System for Training and Evaluation of Laparoscopic Skills (MISTELS) video trainer curriculum and have demonstrated the merit of this system. Six basic skills are scored on time of performance and accuracy. This system has been shown to be highly reliable and reproducible with established construct and predictive validity. Moreover, receiver operator curves have identified passing scores that maximize sensitivity and specificity, both greater than $0.80 .^{9}$

The beneficial influence of training using VR trainers on subsequent laparoscopic procedures has also been reported. Most of the VR simulators incorporate several different exercises, each with tiered levels of difficulty and performance scores based on efficiency, errors, and time of performance. Multiple studies have shown that practicing the VR skills improves the subsequent performance of these same skills. Several studies have reported that VR practice improves in vivo skills during the performance of laparoscopic cholecystectomy in both pigs and humans. ${ }^{10,11}$ In one study comparing video trainer and VR simulators for resident training, $77 \%$ of the trainees preferred the video trainer to the VR simulator. The reasons given for this preference were the use of real instruments and camera systems, better depth perception, and the presence of haptic feedback, which is absent in the VR system. ${ }^{10}$

Recently, the Society of American Gastrointestinal Endoscopic Surgeons (SAGES) has developed a program entitled Fundamentals of Laparoscopic Surgery (FLS). ${ }^{12}$ This is a basic instructional course in the fundamentals of laparoscopic surgery that teaches standardized basic information determined by experts as important. ${ }^{13}$ The FLS program uses CDROMs for teaching cognitive information and the MISTELS system for training and assessing technical skills. This allows hands-on learning of motor skills and structured examinations to document learning and "competence" of both cognitive knowledge and technical skills. The examinations have been rigorously developed and validated over several years of formulation and reiteration such that the test can withstand "high-stakes" scrutiny. The FLS program has undergone beta testing demonstrating that it has appropriate psychometric properties, satisfactory reliability, initial validity evidence, viable administrative procedures, and the capability of setting appropriate "pass-fail" standards.

MIS training in residency is generally adequate for basic laparoscopy - the mean number of laparoscopic cholecystectomies performed by chief residents graduating in 2002 was 75 (American Board of Surgery data). However, in most programs there is limited experience in advanced laparoscopy. The mean cumulative experience for any advanced procedures (combined) was less than 25. This compares to the assessment by experts that the experience necessary for competency in most advanced procedures is between 15 and 35 per procedure. ${ }^{7}$ Furthermore, chief residents generally do not feel adequately trained to perform advanced procedures at the completion of their program. ${ }^{13}$ As a result, more than 80 MIS fellowships have now sprung up around the United States with more than 110 training positions available annually. This is the most popular fellowship currently available in the United States.

Only approximately $15 \%$ of a surgical career is spent in formal surgical training. Continuing education thus becomes very important and can take the form of either deductive reasoning and self-education or formal continuing medical education (CME) programs. General surgery recently experienced a period of haphazard training during the initial laparoscopic cholecystectomy "revolution" of the early 1990s. ${ }^{14}$ At that time, many surgeons adopted laparoscopic techniques with inadequate training, leading to a number of patient injuries that occurred during the "learning curve." In fact, a whole new literature sprang up in general surgery revolving around laparoscopic cholecystectomy-associated bile duct injuries. There were concerns that governmental agencies would determine who could and who could not perform laparoscopic surgery. The American philosopher, George Santayana stated, "Those who cannot remember the past are condemned to repeat it."

Currently, the major needs for retraining of practicing general surgeons in advanced laparoscopic procedures are in the areas of laparoscopic bariatric operations and laparoscopic colectomy (other areas of surgery utilizing minimally invasive technologies are out of the scope of this review). However, it should be noted that the Food and Drug Administration recently mandated that surgeons initiating clinical use of one of the new carotid artery stents be trained on 
a simulator prior to clinical experience. The aim of CME training in surgical techniques should be to eliminate the first very steep portion of the learning curve and to allow the surgeon to start clinical experience in the flatter portion of the curve. Surgical education models in the United States primarily revolve around surgical residency and fellowships. Beyond these formal training opportunities, however, there are no nationally coordinated efforts to provide $\mathrm{CME}$ training from a procedural standpoint. The model adopted during the initial phase of laparoscopic cholecystectomy was that of 1 to 3 day courses using large animals for training. This same model, or variants using cadaver models, continues to be the mainstay for teaching practicing surgeons new procedures. There are a few programs currently that provide hands-on CME courses followed by preceptoring within the institution of the trainee. Other institutions provide "mini-fellowships" that last anywhere from 1 to 6 weeks to allow the trainee time in the clinic, the laboratory, and in the clinical operating room.

There have been very few published reports that support the efficacy of the current postgraduate training models. The group from the Carolinas Medical Center has shown that adding a period of preceptoring in the trainees' own institution (up to 11 cases) after a hands-on CME course leads to significantly increased adoption rates of both laparoscopic splenectomy and laparoscopic incisional herniorrhaphy, compared to course participation without preceptoring. ${ }^{15,16}$ Their data also suggested that surgeons already experienced in advanced laparoscopic procedures prior to taking a 2-day hands-on course teaching a new operation are more likely to adopt the new technique being taught. ${ }^{16}$ There are also anecdotal reports of increased adoption rates following a minifellowship experience; most of these mini-fellowships are currently teaching the technique of laparoscopic bariatric surgery.

There are numerous roadblocks to training established surgeons in advanced MIS techniques. These include available time (both for the trainer and the trainee) and money (trainee), licensing, privileging, and malpractice issues (trainer and trainee), as well as the lack of sound educational models guiding the procedural training of established surgeons. ${ }^{17}$ Certainly, these programs should be based on established principles of training adults and of teaching technical skills (such as those used for teaching musicians). ${ }^{18,19}$

From a personal standpoint I would suggest the following recommendations for "old dogs" desiring to learn "new tricks": The surgeon must decide how important learning the new technique is to him or herself; a weekend CME course is almost certainly not adequate to learn a complex procedure. One's basic skills and two-handed techniques should be optimized; the FLS course may help to improve cognitive and technical skills and to allow comparison of one's skill level to national norms. Mini-fellowships and/or courses plus preceptoring currently seem to provide the best opportunity for effective procedural training. It is probably helpful to train along with a partner or associate to make the initial clinical cases easier to perform. The alternative to formal courses is to hire a fellowship-trained partner and learn from him or her. Certainly, it is imperative not to initiate a clinical program unless or until one feels confident and has had a proctor or preceptor present for at least one case.

In conclusion, surgical residencies are doing a fair job of teaching basic laparoscopic procedures, but are generally inadequate for training advanced laparoscopic procedures. The SAGES FLS program should help assure competency in basic MIS knowledge and skills. MIS fellowships should ultimately provide a supply of trained MIS surgeons; in the meantime, there are established needs for training, particularly for laparoscopic bariatric and colorectal operations. There are numerous hands-on CME courses designed to train practicing surgeons to perform advanced MIS procedures, but there are few data to support the efficacy of these programs. Other procedures and technologies will undoubtedly come along requiring retraining of established surgeons: educators must develop programs to retrain practicing surgeons based on established theories of adult learning and professional technical skills training. These training efforts will likely incorporate new simulator technology in the process.

\section{MAINTENANCE OF CERTIFICATION: DEFINING SURROGATE MEASURES OF COMPETENCY Barbara Lee Bass, M.D.}

As members of a profession, surgeons have a societal obligation to self-regulate and set standards for care. As individual surgeons, we similarly hold a personal obligation to ensure that the care we provide to our patients is based on current surgical knowledge and patient care principles and that the care we provide is properly executed and delivered with autonomy and respect for our patients. The recognition of this professional responsibility was the principle that lead to the creation of certifying boards in American medicine and surgery in the first half of the 20th century. These American boards are unified in setting standards to foster the delivery of high quality health care by ensuring the quality and competency of the physician workforce, as discussed 
above. The ABMS (Table 1), which includes 10 surgical boards, is the umbrella organization unifying these medical and surgical boards.

All member boards have set rigorous standards for achieving initial board certification, defining training requirements, practice experience requirements, assessment of professional standing, and rigorous initial examination requirements. The achievement of board certification is a demonstration that a diplomate has met the high standards of a competent provider in a given specially area. For over 50 years, this initial certification process was the only objective assessment of cognitive knowledge and patient care required of practicing surgeons. Recognizing that this initial competence may not be durable over a 40 or 50 year professional lifespan, the member boards of the ABMS initiated a recertification requirement in 1973. Diplomates would thereafter be required to provide evidence of professional standing, continuing education and pass a secure multiple-choice examination once every 10 years. Although many applauded this effort to reassess a diplomate's knowledge, others criticized the recertification process as a minimal measure of a diplomate's professional abilities-his or her actual competence as a physician or surgeon. For general surgeons, the American Board of Surgery (ABS) recertification examination covers material in all of the essential content areas of general surgery training (Table 3). More than $90 \%$ of diplomates pass the examination at the 10 - and 20-year recertification interval. However, the cohort of diplomates now taking the examination at the 30 -year interval pass the examination at a substantially lower rate; $20 \%$ to $30 \%$ of the examinees fail the exam. Recognizing that most surgeons focus their practices to certain areas within the essential content area, is this comprehensive recertification examination a valid test of knowledge? Are these surgeons in their third decade of practice truly lacking in medical knowledge and patient care skills? Or is the ABS recertification examination too

Table 3. Essential content areas of general surgery training

- Alimentary tract

- Abdomen and its contents

- Breast, skin, and soft tissue

- Endocrine system

- Head and neck surgery

- Pediatric surgery

- Surgical critical care

- Surgical oncology

- Trauma/burns

- Vascular surgery broad in scope? Or does it poorly test their knowledge in their actual areas of practice?

In recent years, public scrutiny of the health care system and of the quality of care provided by physicians and surgeons has increased dramatically. Physician competency has been challenged, albeit perhaps unfairly. Nonetheless, the member boards of the ABMS have taken the opportunities provided by such scrutiny to reassess our own standards for objective appraisal of how we critique our professional activities. In 1999, as a first step in this selfappraisal, the ABMS defined the core components of six competencies (Table 2) to define those aspects of being a physician and surgeon that are essential to high-quality patient care (see first section of this article). Applying metrics to these competencies, however, proved to be more difficult. Hence, the member boards have sought to develop a process that can utilize surrogate measurable criteria to assess objectively evidence of competency in practice on a continuous basis. Crafted over the last 3 years, the Maintenance of Certification (MOC) program was unanimously endorsed by the ABMS in 2002. In MOC all member boards have committed to agree to evolve from periodic recertification programs to MOC programs. The programs are to be initiated in 2005 for all new diplomates and on a rolling basis for diplomates whose certificates are expiring in the old recertification process.

The ABMS has defined four components essential to all MOC programs. Each board must develop criteria to assess (1) professional standing, (2) evidence of lifelong learning and self-assessment, (3) cognitive expertise, and (4) practice performance and assessment. Each member board can stipulate the precise methods to be utilized for each of these four components provided the criteria are consistent with the framework endorsed by the ABMS at large. The ABS has been carefully considering these metrics for the last 2 years. Some of the criteria have been formulated while others remain in evolution. The remainder of this report will summarize the current framework of the MOC program in general surgery of the ABS (from Surgery ABo, maintenance of certification information file). The unresolved issues in each of the four required areas are also being addressed.

\section{Part 1: Assessment of Professional Standing}

The ABS will require all diplomates to maintain an unrestricted license to practice medicine; loss of licensing will result in forfeiture of the ABS certificate. Verification of clinical privileges and professional standing will be required from chiefs of surgery or chairs of credentials committees at institutions 
where surgeons practice, every 3 years. While these criteria may seem straight forward, application of these requirements may prove challenging for some diplomates. The ABS is considering how to allow surgeons with professional activities restricted to administrative, research, educational, or other activities without clinical privileges to meet this requirement. Should these surgeons maintain active certificates the same way surgeons in practice do? Should the certificate provided to surgeons in these capacities reflect this difference?

\section{Part 2: Demonstration of Lifelong Learning and Self-Assessment}

ABS diplomates will be required to complete 50 hours of continuing professional development (CPD) education, 30 of which must be Category 1 each year. Proof of CPD completion will be required every 3 years. Furthermore, diplomates will be required to complete a self-assessment examination every 3 years after certification. The testing tools for this selfassessment are not fully defined. Materials that will meet the requirements include American College of Surgeon programs such as the Surgical Education and Self-Assessment Program, the testing modules in the Fournal of the American College of Surgeons, and other publications. The ABS is partnering with the American College of Surgeons to inventory materials that could be of value to diplomates in both the selfeducation and assessment programs. Issues that remain unresolved regarding Part 2 include the scope and content of the CPD and testing. Should diplomates be required to maintain current knowledge in all the essential content areas of general surgery? Should they be allowed to read and test in areas only relevant to their actual practices? Should the ABS craft a modular MOC education and assessment program, allowing diplomates to complete a "core" content area, perhaps covering perioperative management and areas of general surgery essential to the public welfare in the event of national emergency, and then add on modules precisely relevant to their practices? In many respects, the program with greatest public credibility is the latter-patients want their doctors to be knowledgeable in their actual areas of practice, not in areas where they have no clinical activity.

\section{Part 3: Demonstration of Cognitive Expertise}

ABS diplomates will be required to take a secure examination once in every 10-year MOC cycle. At the present, the exam is anticipated to be a multiplechoice computer-based examination. As in Part 2, unresolved issues include definition of the scope of the content of the examination-broad or focused, comprehensive or modular-and again the question of truth in labeling with the certificate remains undetermined. Should all certificates look the same, regardless of the examination content?

\section{Part 4: Practice Performance Assessment}

For the ABS, the means to assess this requirement remains under discussion. Ideally a method of outcomes assessment that is scientifically and educationally valid will be developed to allow diplomates to review their own practices and in so doing improve the quality of care they practice. The ABS recognizes, however, that measures to assess outcomes of practice remain rudimentary at best. The Veterans Administration National Surgical Quality Improvement Program (NSQIP) is the best developed outcome system; however, even this program allows measurement only of outcomes of surgical services rather than individual surgeons. This program has now been validated in private sector hospitals in an Agency for Healthcare Research and Quality-funded American College of Surgeons sponsored multisite trial, and the ACSNSQIP Program was opened to enrollment of private sector hospitals around the country in October 2004. One could envision that diplomates practicing in NSQIP hospitals, which have the only risk-adjusted outcomes system in existence, would have access to their own outcomes data and as such their review of these data could meet the MOC program requirements. However, this program is currently available to a very limited number of diplomates. The ABS is considering other programs that diplomates could participate in to meet this requirement, including cooperative groups, surgical society registries, and others.

The ABS, like other ABMS boards, does not wish to hold and review outcomes. In fact, liability aspects of collecting patient and physician outcome data are problematic for ABMS boards and are the topic of ongoing debate. The ABS does, however, desire to create a system that will allow diplomates to participate in outcomes assessment programs to accurately and openly assess the results of their surgery so that areas for improvement can be identified. Although trials of surgeon self-reporting systems in the past have not been successful, possible avenues for selfreporting and benchmarking against standards are being considered. The American College of Surgeons is developing a personal digital assistant-based case reporting system for fellows. Surgeons could enter clinical data on their patients and then subsequently benchmark their own performance on this selfreported data relative to nurse-collected data in the 
NSQIP. Although self-reported data would be used, this would serve as a starting point to allow surgeons an opportunity to get a fair appraisal of their own results. Fundamentally believing that our diplomates do wish to provide the highest quality care and do wish to identify areas for improvement in their own practice, this confidential benchmarking program could be a valuable first step in practice assessment. Diplomates who find themselves with outcomes below standard would be stimulated to pursue educational programs to foster improvement in the care they provide.

In summary, the goal of the ABS MOC program is to document and improve the overall quality of the care provided by a surgeon throughout a lifetime of practice. The goal is not to identify the "bad apple" surgeon. The goal is to encourage individual surgeons to commit to lifelong education and scrutiny of their own outcomes, because it is the right thing to do for patients. Surrogate measures for competence developed for the ABS MOC program will need to evolve in the years ahead. The lessons learned from our initial efforts will drive the process and guide us as we establish the systems to continuously improve the self-appraisal systems we as a profession must own.

\section{REFERENCES}

1. Nahrwold DL. Presidential address: toward physician competency. Surgery 1999;126:589-593.

2. Nahrwold DL. The competence movement: A report on the activities of the American Board of Medical Specialties. Bull Am Coll Surg 2000;85:14-18.

3. Nahrwold D. The best surgical thinking: Surgical competence. What's New in ACS Surgery (online publication). 2004.

4. Nahrwold DL. Why the 6 general competencies? Surgery 2004;135:4-5.

5. Neufeldt VE. Webster's New World College Dictionary. New York: Simon and Schuster, 1997.
6. Villegas L, Schneider BE, Callery MP, Jones DB. Laparoscopic skills training. Surg Endosc 2003;17:1879-1888.

7. Park A, Witzke D, Donnelly M. Ongoing deficits in resident training for minimally invasive surgery. J GASTROINTEST SURG 2002;6:501-507.

8. Feldman LS, Hagarty SE, Ghitulescu G, et al. Relationship between objective assessment of technical skills and subjective in-training evaluations in surgical residents. J Am Coll Surg 2004;198:105-110.

9. Fried GM, Feldman LS, Vassiliou MC, et al. Proving the value of simulation in laparoscopic surgery. Ann Surg 2004; 240:518-525.

10. Hamilton EC, Scott DJ, Fleming JB, et al. Comparison of video trainer and virtual reality training systems on acquisition of laparoscopic skills. Surg Endosc 2002;16:406-411.

11. Seymour NE, Gallagher AG, Roman SA, et al. Virtual reality training improves operating room performance: Results of a randomized, double-blinded study. Ann Surg 2002;236: 458-463.

12. Peters JH, Fried GM, Swanstrom LL, et al. Development and validation of a comprehensive program of education and assessment of the basic fundamentals of laparoscopic surgery. Surgery 2004;135:21-27.

13. Rattner DW, Apelgren KN, Eubanks WS. The need for training opportunities in advanced laparoscopic surgery. Surg Endosc 2001;15:1066-1070.

14. Soper NJ. SAGES and surgical education: Assuring that history does not repeat itself. Surg Endosc 2001;15:775-780.

15. Heniford BT, Backus CL, Matthews BD, et al. Optimal teaching environment for laparoscopic splenectomy. Am J Surg 2001;181:226-230.

16. Heniford BT, Matthews BD, Box EA, et al. Optimal teaching environment for laparoscopic ventral herniorrhaphy. Hernia 2002;6:17-20.

17. Rogers DA, Elstein AS, Bordage G. Improving continuing medical education for surgical techniques: Applying the lessons learned in the first decade of minimal access surgery. Ann Surg 2001;233:159-166.

18. Kaufman HH, Wiegand RL, Tunick RH. Teaching surgeons to operate-principles of psychomotor skills training. Acta Neurochir (Wien) 1987;87:1-7.

19. Eva KW. Stemming the tide: Cognitive aging theories and their implications for continuing education in the health professions. J Contin Educ Health Prof 2003;23:133-140. 